\title{
RECENT PROGRESS AT LBNL ON CHARACTERIZATION OF LASER WAKEFIELD ACCELERATED ELECTRON BUNCHES USING COHERENT TRANSITION RADIATION*
}

\author{
G. Plateau ${ }^{\dagger}$, E. Esarey ${ }^{\ddagger}$, C. G. R. Geddes, W. P. Leemans ${ }^{\ddagger}$, N. Matlis, \\ C. B. Schroeder, J. van Tilborg, Cs. Tóth \\ Lawrence Berkeley National Laboratory (LBNL), Berkeley, CA 94720, USA
}

\section{Abstract}

At LBNL, laser wakefield accelerators (LWFA) can now produce ultra-short electron bunches with energies up to $1 \mathrm{GeV}$ [1]. As femtosecond electron bunches exit the plasma they radiate an intense burst in the terahertz range $[2,3]$ via coherent transition radiation (CTR). Measuring the CTR properties allows non-invasive bunchlength diagnostics [4], a key to continuing rapid advance in LWFA technology. Experimental bunch length characterization for two different energy regimes through bolometric analysis and electro-optic (EO) sampling are presented. Measurements demonstrate both shot-to-shot stability of bunch parameters, and femtosecond synchronization between the bunch, the THz pulse, and the laser beam. In addition, this method of CTR generation provides $\mathrm{THz}$ pulses of very high peak power suitable for applications. Recent results reveal LWFA to be a promising intense ultrafast $\mathrm{THz}$ source.

\section{BOLOMETRIC ANALYSIS OF LOW ENERGY ELECTRON BUNCHES}

Currently, one principal challenge encountered in achieving LWFA stability is the control of the injected electrons. One strategy to address this issue is to decouple injection and acceleration (allowing better control over plasma parameters) by externally injecting electrons into a preformed plasma channel. Simulations indicate such injection preserves the energy spread [5]. Thus, the controlled injection of ultrashort bunches with low emittance and low divergence may produce stable $0.1 \%$ energy spread beams at $\mathrm{GeV}$ energies.

At the LOASIS Program, a low electron energy regime $(<1 \mathrm{MeV})$ is presently being studied for its stability and possible suitability as injector (hundred keV-level energy spread and pointing variation near $2 \mathrm{mrad}$ ). To be phase trapped in the next stage the beam needs to be short enough (fraction of a plasma wavelength) and so its length must be characterized.

Electron bunches generated by the LWFA technique emit coherent transition radiation (in the $\mathrm{THz}$ range) when they

\footnotetext{
* Work supported by US DoE Office of High Energy Physics under contract DE-AC03-76SF0098 and DARPA

${ }^{\dagger}$ Also at École Polytechnique, Palaiseau 91128, France

${ }^{\ddagger}$ Also at University of Nevada, Reno, Nevada 89557, USA
}

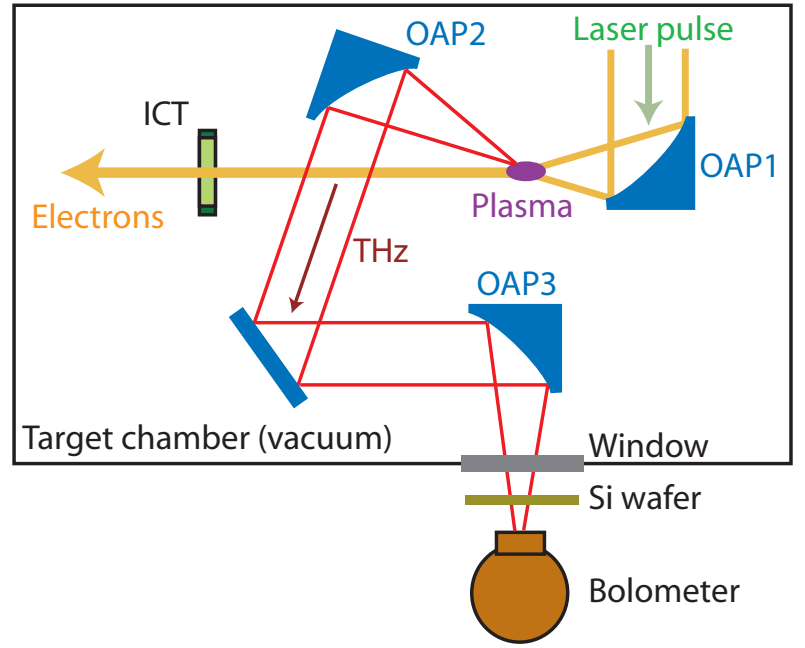

Figure 1: Setup for measurement of the THz pulse energy generated by a $1 \mathrm{MeV}$ electron beam. A 6-inch effective focal length off-axis parabola (OAP) is used to collect partially the $\mathrm{THz}$ pulse emitted by transition radiation. A 7inch OAP is used to focus it back into the bolometer aperture.

cross the plasma-vacuum boundary. Characterization of the $\mathrm{THz}$ pulse allows retrieval of the temporal charge distribution of the produced bunch and thus serves as bunch diagnostic.

The amount of transition radiation produced by a $1 \mathrm{MeV}$ beam is too small to be characterized by classical EO techniques. Here a technique (Fig. 1) is presented using a bolometer $\left(\lambda \simeq 10-200 \mathrm{~cm}^{-1}\right)$ [6,7]. While a bolometer provides an exceptionally sensitive measurement of the $\mathrm{THz}$ energy, it does not normally yield information about temporal electric profile. However, by measuring the ratio between radiation collected with and without a spectral filter the electron bunch duration can be estimated [3,7]. Indeed, the general expression for CTR spectrum depends on the bunch length $\left(\sigma_{z}\right)$.

Two different materials were used to filter the spectrum, Teflon (1/4" thick) and Fluorogold (0.5 mm thick), with spectral cut-offs at $\sim 2 \mathrm{THz}$ and $\sim 1 \mathrm{THz}$, respectively. From the bolometer measurements with and without the filters, the bunch length was determined to be $\sigma_{z} \sim 300+$ $590,-190$ fs using Teflon, and $\sigma_{z} \sim 210+120,-80$ fs us- 
ing Fluorogold. (Teflon has a less abrupt cut-off frequency, reducing its precision.) These lengths agree with bunch lengthening due to space charge in a plasma density downramp [8]. Recent simulations [9] indicate a $1 \mathrm{MeV}$ bunch is $\sim 10 \mu \mathrm{m}$ long at production and lengthens to $\sim 50 \mu \mathrm{m}$ ( $\sim 177 \mathrm{fs})$ within the down-ramp. Hence, injection into a second plasma channel is achievable by using a sharp transition between the two stages.

\section{ELECTRO-OPTIC IMAGING OF TERAHERTZ PULSES}

Previous work [10,11] showed LWFA bunch durations of $<50$ fs $(15 \mu \mathrm{m})$ with high intensity peak $\mathrm{THz}$ electric field up to $0.4 \mathrm{MV} / \mathrm{cm}$ for beams in the range $10-100 \mathrm{MeV}$. However, theory [3] indicates that optimization may yield even higher $\mathrm{THz}$ electric fields. Looking at the spatiotemporal profile of the electric field, aberrations have been observed that result in imperfect focusing. This has motivated the construction of a new setup that has allowed for significant improvements in the focal spot quality.

\section{Spatiotemporal Profile}

The new setup which includes several motorized optics, has addressed these optical issues and a single shot 2-D imaging technique [11] used to yield knowledge of the $\mathrm{THz}$ spot size and spatial structure. By combining a collimated and linearly polarized probe beam with the $\mathrm{THz}$ pulse at focus in an electro-optic (EO) crystal (ZnTe or GaP) local rotation of the polarization is induced in the probe beam. The THz pulse (low frequencies) acts as an electrical bias on the crystal. The probe beam continues through a second polarizer set for a maximum extinction of the initial linear polarization of the probe, and a CCD camera records the remaining beam.

By scanning the delay between the $\mathrm{THz}$ pulse and the probe, spatial slices of the $\mathrm{THz}$ pulse are measured. Although additional analyses show a small level of remaining aberration (coma) in the terahertz beam, the temporally averaged mode displays a near-Gaussian shape (Fig. 2). This mode corresponds to what would be observed by a terahertz-sensitive-camera (summation of the movie frames). The beam at focus has a radius (standard deviation) of $\sim 0.26 \mathrm{~mm}$.

\section{Electric Field Measurement}

Different EO techniques [10] are available to measure the temporal electric field profile of the $\mathrm{THz}$ pulse either in a single-shot or scanning mode. Most of them are based on the assumption of a homogeneously distributed electric field over the focal spot, which implies having both a perfect source and a perfectly aligned optical line. The 2-D imaging technique previously introduced confirms this alignment and gives an estimate for the averaged electric field.

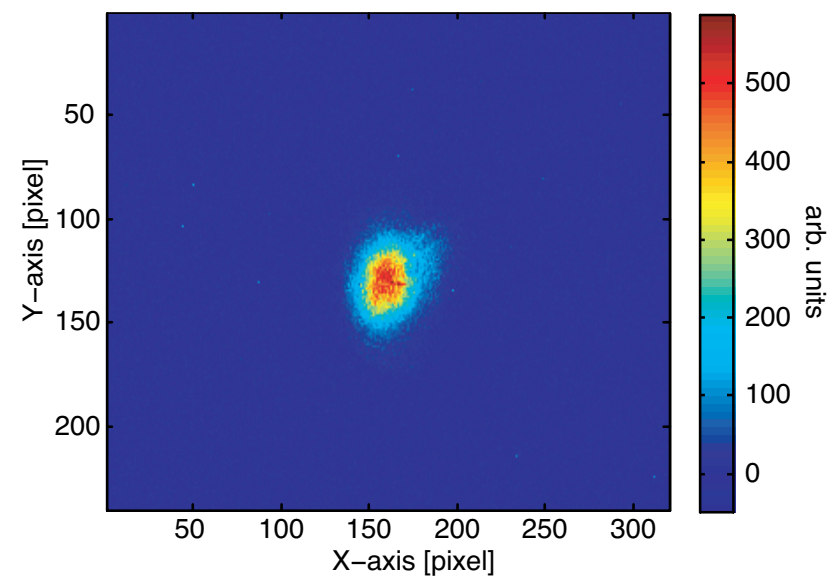

Figure 2: Imaging of a focused $\mathrm{THz}$ pulse (CTR) emitted by a LWFA. CCD camera averaged signal for different delays between the CTR emitted when electrons exit the plasma and the $43 \mathrm{fs}$ long probe beam.

CTR is emitted along a radially polarized cone of light defined approximately by the angle $\sim 1 / \gamma$, where $\gamma$ is the relativistic factor of the produced electron bunch. A small portion of this cone is collected by an $\operatorname{OAP}\left(f_{\#}=2\right)$. Since the parabola only collects a small solid angle $(0.178 \mathrm{sr})$ the collected $\mathrm{THz}$ beam is considered to be linearly polarized. The Pockels effect happening in the EO crystal $(\mathrm{GaP})$ used for this experiment depends [11] on the relative orientation of one of its principal axes to the linear polarization of both the $\mathrm{THz}$ beam to study and the probe beam. In order to optimize the rotation of the probe bream's polarization, these last two were parallel to each other but perpendicular to the concerned crystal axis.

$$
\frac{I_{T}}{I_{T}^{0}}=\frac{1-\cos (\Gamma)}{2}
$$

The normalized transmitted intensity through the analyzer is a function (Eq. 1) of the phase retardation introduced in the probe, $\Gamma=\Delta n \frac{\omega \cdot L}{c}$ where $\Delta n \propto \vec{E}_{\mathrm{THz}}$ is the difference of refraction indexes induced by Pockels effect, $\omega$ is the frequency of the probe beam and $L$ is the thickness of the crystal. A calibration using a $\lambda / 2$ plate gives a correspondence between the angle of rotation of the probe's polarization and the transmitted intensity. Taking into account both crystal effects (surface losses, $\mathrm{x} 2$, and dispersioninduced decrease, $\mathrm{x} 2$ ) and spectral absorption (30\%) of the high density polyethylene window (used as vacuum window), the averaged peak electric field is calculated to be $\sim 102 \mathrm{kV} / \mathrm{cm}$ before entering the imaging optical system.

\section{OPTIMIZING THE LWFA AS A COMPACT, HIGH FIELD THZ SOURCE}

Coherent transition radiation can be used as an electron bunch duration measurement. THz electric fields produced by LWFA are also exceptionally strong and are promising 
to be a useful source of high field $\mathrm{THz}$ pulses. The important beam-plasma parameters that determine the shape of the spectrum and hence the amount of energy per $\mathrm{THz}$ pulse, are the electron bunch charge, length $\left(\sigma_{z}\right)$, kinetic energy ( $E_{c}$, weak dependence), the plasma boundary size, and the collection angles.

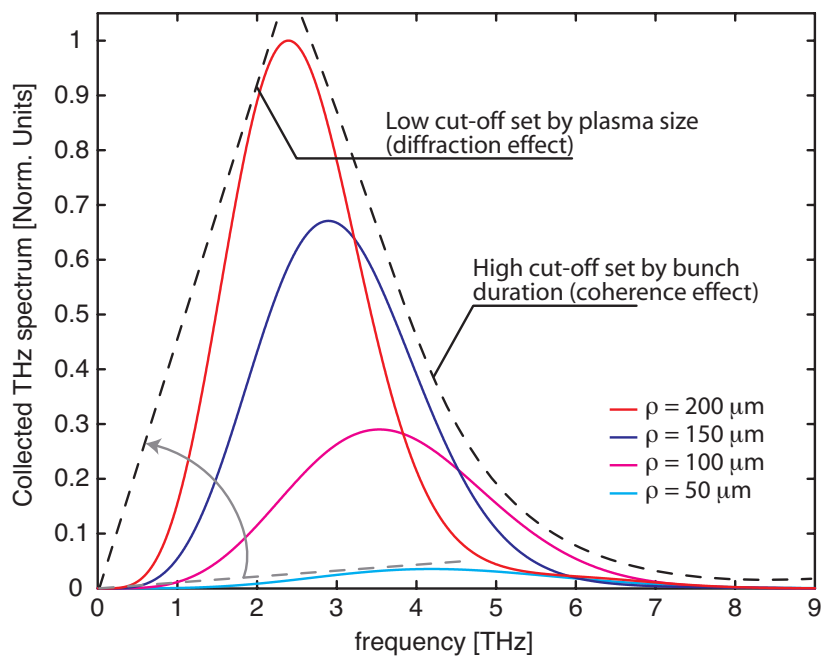

Figure 3: Predicted spectra ( $0.5 \mathrm{nC}, \sigma_{z}=15 \mu \mathrm{m}, E_{c}=45$ $\mathrm{MeV})$ of CTR collected by a 6-inch OAP (19 ${ }^{\circ}$ off-axis) for different plasma transverse boundary sizes $(\rho)$. The amount of emitted energy is strongly affected by the transverse boundary size of the emitting region.

Two phenomena strongly dominate the CTR in spectral domain: diffraction effects due to the limited size of the plasma and coherence effect due to the longitudinal distribution of the electron beam (Fig. 3).

Increasing the plasma boundary size can be achieved by sending a pre-ionizing pulse to enlarge the plasma in front of the main pulse. A preliminary experiment has been performed at the LOASIS Program using such coupled beams, and the enhancement of the THz energy generated by CTR has been observed via a bolometric measurement (Fig. 4).

Predictions for beams of higher charge $(\sim 1 \mathrm{nC})$ produced in a pre-ionized channel (up to $200 \mu \mathrm{m}$ ) and collected by a 6-inch OAP are above $30 \mu \mathrm{J}$ per pulse. Moreover, the weak electron energy dependence allows possibility of building a compact LWFA (smaller laser) dedicated to $\mathrm{THz}$ production with a bigger solid angle of collection. Potentially, THz pulses of up to $0.7 \mathrm{~mJ}$ centered around $2 \mathrm{THz}$ could be produced, opening new fields of application.

\section{REFERENCES}

[1] W. P. Leemans et al., Nature Physics 2, (2006) 696.

[2] W. P. Leemans et al., Phys. Rev. Lett. 91, (2003) 074802.

[3] C. B. Schroeder et al., Phys. Rev. E 69, (2004) 016501.

[4] J. van Tilborg et al., Phys. Rev. Lett. 96, (2006) 014801.

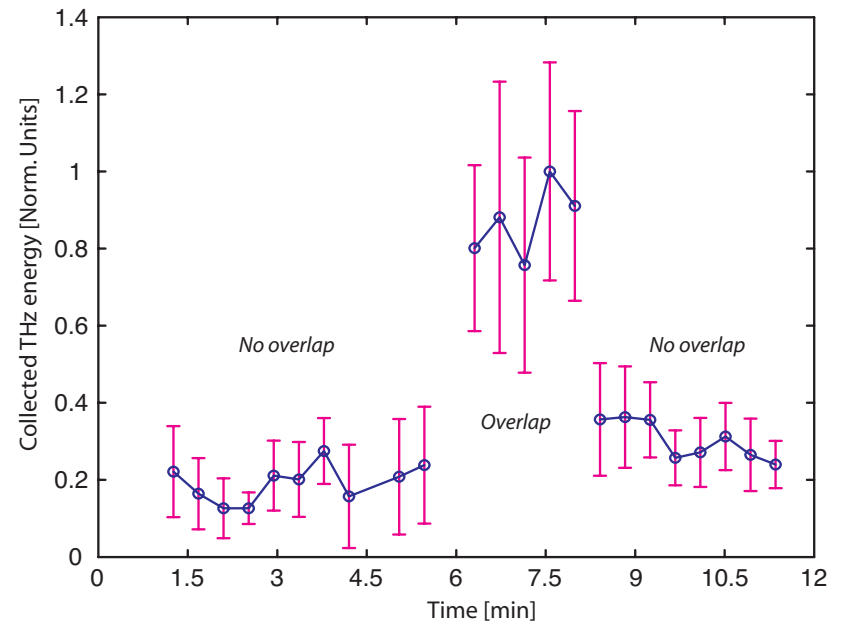

Figure 4: Enhancement $(>3)$ of the total amount of $\mathrm{THz}$ energy collected observed when the main pulse (acceleration) is preceded with a pre-ionizing beam (on/off experiment).

[5] B. A. Shadwick et al., Bull. Am. Phys. Soc. Plasma Physics Meeting 47, (2005) 213.

[6] P. L. Richards, J. Appl. Phys. 76, (1994) 1.

[7] W. P. Leemans et. al., Phys. of Plasma 11, (2004) 2899.

[8] G. Fubiani et al., Phys. Rev. E 73, (2006) 026402.

[9] C. G. R. Geddes et al., "Stable Electron Beams With Low Absolute Energy Spread From a Laser Wakefield Accelerator With Plasma Density Ramp Controlled Injection", PAC'07, Proceedings, June 2007.

[10] J. van Tilborg, Ph.D. thesis, Technische Universiteit Eindhoven (2006).

[11] J. van Tilborg et al., Opt. Lett. 32, 3, (2007) 313.

[12] Q. Chen et al., JOSA B 18, 6, (2000) 823. 